\title{
A search and improvement of the geometric parameter betatron injector
}

\author{
Andrey Kolomeytsev ${ }^{1, *}$, and Mikhail Shtein ${ }^{2}$ \\ ${ }^{1}$ National Research Tomsk Polytechnic University, Department of Electronic Engineering, 634050 \\ Tomsk, Russia \\ ${ }^{2}$ National Research Tomsk Polytechnic University, Research and Production Laboratory "Radiation \\ Inspection and Industrial Safety Systems", 634050 Tomsk, Russia
}

\begin{abstract}
The article describes the search for optimal geometric parameters of the betatron injector. First of all, we determine parameters that influence the injection efficiency. Next, we introduce the coefficient that directly connects the geometric parameters of the injector with capture efficiency. The computer model of the betatron injector is recreated. In the injector region, there are forces that are equal in magnitude and direction to forces in a real betatron. We simulate different parameter combinations. After processing the results, tolerances are obtained for the selected parameters. The results of this work can be used as recommendations for the development of design documentation for new three-electrode injectors for betatrons. The research method can be used to study the efficiency of other injector designs, as well as to search for new design alternate.
\end{abstract}

\section{Introduction}

The injector is the key betatron component. Injector parameters determine the maximum number of electrons that can be captured by the magnetic field of a betatron. However, the optimal geometric parameters of an injector have not been defined up to the present day. The manufacturing tolerance of these parameters is still undefined either. From the large number of electrons emitted by a cathode injector, only a small percent is captured by the accelerating field. There are many reasons behind this phenomenon that are confirmed experimentally. These include the following:

- Insufficient injection energy;

- Low electron space-charge density;

- Low synchronization accuracy between the injection and accelerating flux;

- Insufficient stability of the voltage pulse generation;

- Non-optimal geometry and relative position of electrodes [1-5].

This research work considers how geometrical parameters of the injector affect the coefficient of its current transmission.

The active part of the capture process takes approximately one microsecond. During this period of time, the initial electron velocity (injection voltage) is adjusted to the magnetic

\footnotetext{
*Corresponding author: aak65@tpu.ru
} 
field magnitude. Meanwhile, $(4 \ldots 5) \cdot 10^{12}$ electrons are injected into the interpole space. A betatron only captures $(1 \ldots 1.5) \cdot 10^{10}$ particles, in other words, one electron out of $300 \ldots 500$. The ratio of the captured electrons to the injected ones is capture efficiency [6-8].

\section{Materials and methods}

Betatron electromagnet has an annular gap and is supplied with an alternating current. The accelerating chamber is set in the annular gap. The chamber has a high vacuum (10-6 mm. $\mathrm{Hg}$. Art.) inside. The injector is mounted in one of the accelerating chamber nipples.

Figure 1 shows the image of the injector and its geometrical parameters.
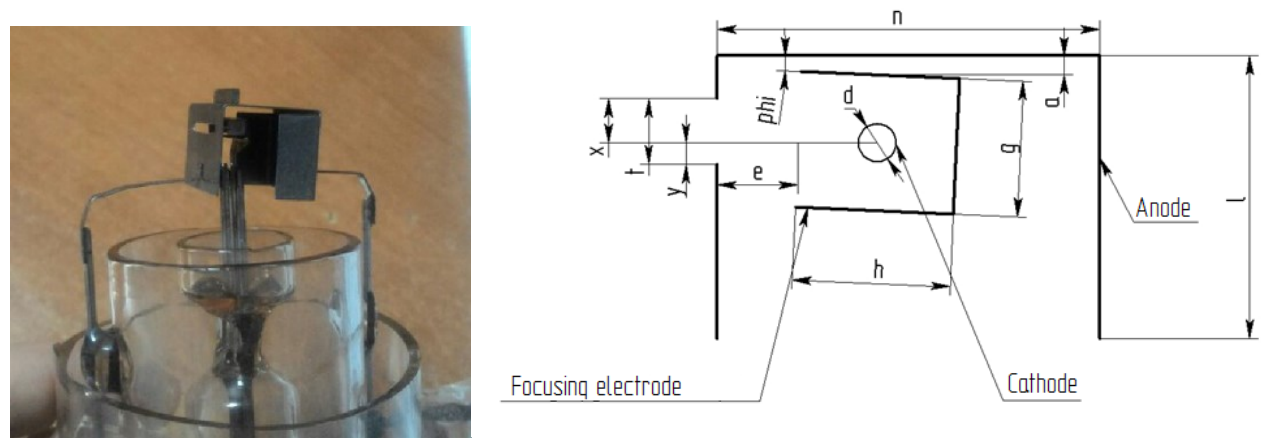

Fig. 1. The injector and a schematic drawing of labeled parameters.

The parameter $a$ determines the electrical injector stability and shows the distance between the center of the top plate of the focusing electrode and the anode.

The parameter e shows the distance between the focusing electrode and the anode gap.

The parameter $f$ (not shown in the figure) is called misalignment and is defined as follows. Through the middle of the focusing electrode a projection extends perpendicular to the gap line. Next, we measure the distance from the line to gap edges $x$ and $y$, and calculate the misalignment $f$ according to formula (1). The displacement to the betatron center is positive.

$$
f=\frac{x-y}{2}
$$

The angle of the focusing electrode against the anode $\varphi$ is the angle between the top of the anode and the focusing electrode plate. A counterclockwise rotation is positive.

The parameter $t$ is the anode gap width. Electrons leave the injector through it.

The parameters $h$ and $g$ determine the width and height of the focusing electrode, respectively. It is also necessary to take into account the horizontal $s H$ and vertical $s V$ displacement of cathode relative to the focusing electrode center. Of particular importance is the parameter $\mathrm{d}$ that describes the cathode diameter.

The dependence of the coefficient of the current transmission on the geometric parameters of the injector is determined by multi-objective optimization. To do this, we set the values of all the parameters to certain (factory) values, then we change only one of them and observe how it affects the result.

Currently, there are several theories that describe the electron capture mechanism in acceleration. However, they only give a qualitative description. So, now it is not possible to create an analytic model to directly connect the geometric parameters of the injector with capture efficiency. However, there is a parameter that can be included in the analytic model to make capture efficiency easy to measure. This is called the coefficient of the current transmission of the injector. 


$$
K_{\mathrm{T}}=\frac{I_{L}}{I_{A}+I_{L}}=\frac{I_{L}}{I_{K}}
$$

As be seen from the formula (2), the maximum injector efficiency is achieved when the coefficient of the current transmission equals. Based on this statement, the main purpose of this study is to define tolerances of the injector parameters, at which the value tends to 1 [9].

In this case, the current transmission coefficient is the ratio of the total charge of the electrons emerging from the injector to the total charge of the electrons that overcome the accelerating gap of the injector, some of which face the anode.

$$
K_{\mathrm{T}}=\frac{N_{L}}{N_{A}+N_{L}}=\frac{N_{L}}{N_{K}}
$$

where - $N_{L}$ - is the total charge of the electrons that overcome the accelerating gap of the injector, $N_{\mathrm{K}}-$ is the total charge of the electrons emerging from the injector.

\section{Results and discussion}

Inside the injector, an electron moves under the influence of the electrostatic field and the magnetic field. The magnetic field shifts an electron to the equilibrium orbit. The intensity of the electrostatic field $\mathbf{E}$ at any point is a physical quantity defined by the force acting on a charge placed at that point of the field. Intensity $\mathbf{E}$ coincides with the direction of the force acting on the charge.

Figure 2 shows the electric field potential in the central section of the injector.

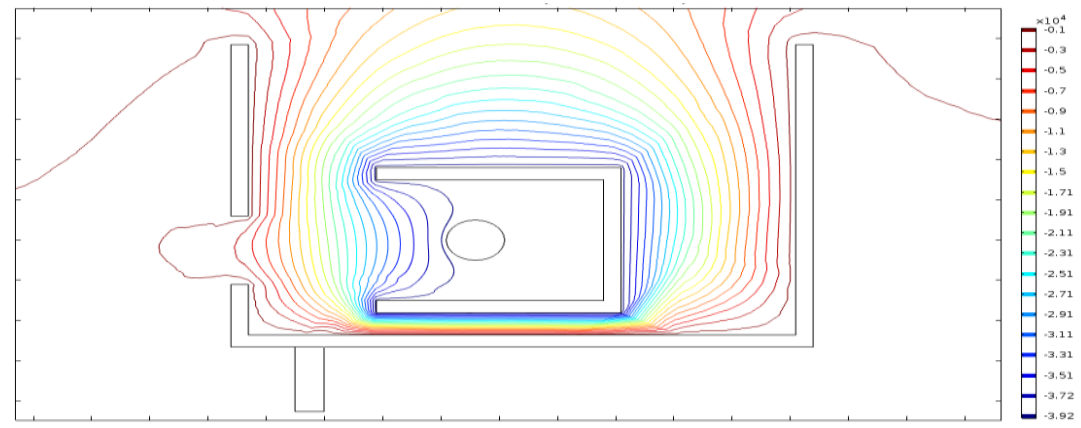

Fig. 2. Equipotential lines of the electric field.

At the injection moment, contractor biases the magnetic field in such a way as to dislocate the equilibrium orbit close to injector. It allows avoiding significant radial oscillation at the acceleration beginning. There is an empirical formula (4) for the calculation of the magnetic induction at the any radius of interpolar space. It allows calculating the required induction of the magnetic field to hold the electrons of certain energy [10].

$$
\left(W\left(W+2 E_{0}\right)^{\frac{1}{2}}=300 B_{0} R_{0}\right.
$$

where $R_{0}$ - is the radius of the equilibrium orbit, $\mathrm{m} ; B_{0}-$ is the magnetic induction at the equilibrium orbit, $\mathrm{T} ; E_{0}-$ is the electron rest energy, $\mathrm{MeV}$.

From the knowledge of radius, electron rest energy and the fact that leaving electrons have an energy of about $40 \mathrm{keV}$, we can calculate the value of the magnetic induction at the injector. 


$$
B_{0}=\frac{\left(W\left(W+2 E_{0}\right)^{\frac{1}{2}}\right.}{300 R_{0}}=0.0064 T
$$

The next step is to simulate the motion of a charged particle in an electromagnetic field. Modeling is based on the calculation results of the magnetic and electric fields. The calculation is only possible after the imposition of boundary conditions on the task. It is important to note that the problem has a number of assumptions and simplifications. First, the electron injection is carried out at a thermionic emission cathode with heating. The average energy of the electrons leaving the metal surface with a thermionic emission $E_{T E}=0.17 \mathrm{eV}$. Second, the electrons can leave the cathode surface in any direction. We set the initial velocity vector directed normal to the surface of the cathode. Third, at this stage of the work, we do not take into account the Coulomb interaction of the electrons with each other because of low charge density.

Certain electron portion falls onto focusing electrode or anode (Figure 3). It means so geometrical parameters of the injector exist that each or the most electrons is able to leave the injector. The determination of such a configuration makes it possible to increase the number of successfully injected electrons and the effective cathode area.

a)

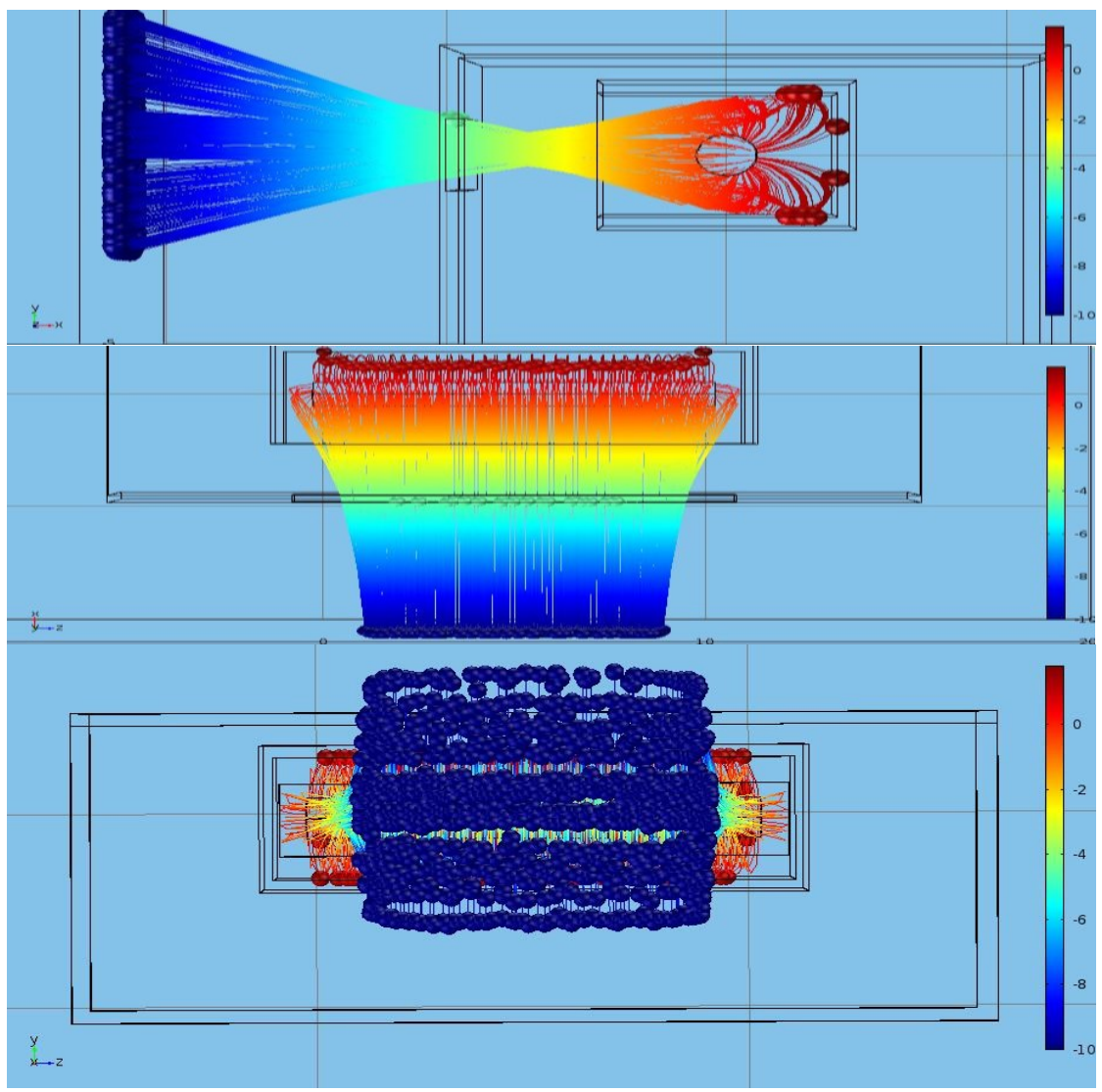

Fig. 3 ( $a-X Y$ plane, $b-X Z$ plane, c - YZ plane). Injected electron trajectories.

We have simulated acceleration with different parameter values. The results are shown in the Table 1. Table 1 describes how to set injector elements to achieve high injection efficiency. 
Table 1.The summary tabulation of the injector parameters.

\begin{tabular}{|c|c|c|c|}
\hline Parameter & Quantity & $\begin{array}{c}\text { Upper } \\
\text { limit }\end{array}$ & $\begin{array}{c}\text { Lower } \\
\text { limit }\end{array}$ \\
\hline $\mathrm{a}, \mathrm{mm}$ & 0.7 & 0.9 & 0.55 \\
\hline $\mathrm{e}, \mathrm{mm}$ & 2.2 & 3.0 & - \\
\hline $\mathrm{f}, \mathrm{mm}$ & 0.15 & 0.4 & 0 \\
\hline $\mathrm{t}, \mathrm{mm}$ & 1.75 & 1.9 & 1.5 \\
\hline$\varphi, \mathrm{deg}$ & 0 & 3.5 & 2.5 \\
\hline
\end{tabular}

\begin{tabular}{|c|c|c|c|}
\hline Parameter & Quantity & $\begin{array}{c}\text { Upper } \\
\text { limit }\end{array}$ & $\begin{array}{c}\text { Lower } \\
\text { limit }\end{array}$ \\
\hline $\mathrm{g}, \mathrm{mm}$ & 3.6 & 3.8 & 3.4 \\
\hline $\mathrm{h}, \mathrm{mm}$ & 4.2 & - & 3.9 \\
\hline $\mathrm{sH}, \mathrm{mm}$ & -0.4 & -0.2 & -0.5 \\
\hline $\mathrm{sV}, \mathrm{mm}$ & 0 & 0.4 & -0.3 \\
\hline & & & \\
\hline
\end{tabular}

\section{Conclusion}

In this study, we have identified the most important parameters, which have an effect on electron injection. A computer model is used to simulate some of the processes inside the injector. The paper has shown how the geometrical parameters of the injector affect the coefficient of the current transmission. We have also calculated the optimal values of these parameters and assigned the appropriate tolerances to them. This set of parameters allows us to obtain an injector whose current transmission will always be $K_{T} \geq 0.9$. The results of this work can be used as recommendations for the development of design documentation for new three-electrode injectors for betatrons. The research method can be used to study the efficiency of other injector designs, as well as to search for new design alternate.

\section{References}

1. J. T. Seeman, Proc. of B FACTORIES 233 (1998)

2. H. Wiedemann, Particle Accelerator Physics I - Basic Principles and Linear Beam Dynamics (Springer) ISBN 3-540- 64672-X

3. V. Moskalev and V Chaklov Betatrons (Tomsk: TPU Publish House, 2009) in Russian

4. M. Aiba et al., Phys. Rev. ST Accel. Beams 18, 020701 (2015)

5. D Greene, The British journal of radiology 34(398) (1961)

6. V Moskalev and G Sergeev The Induction Electron Accelerator - Betatron (Tomsk: TPU Publish House, 2012) in Russian

7. Rychkov M. M. et al. J. of Phys.: Conf. Ser. 881(1), (2017)

8. I. Zatonov, M. Shtein, MATEC Web of Conf. 48 (2016)

9. Shestak A. P. et al., IOP Conf. Series.: Mat. Sci.\&Eng., 81(1), (2015)

10. A. Sedoi and M. Shtein, Instruments and experimental techniques 19(5), (1976) 